\title{
The Future of Direct Electron Detection in Cryo-TEM
}

Gijs van Duinen ${ }^{1}$, Lingbo Yu ${ }^{1}$, Erik Franken ${ }^{1}$, Maarten Kuijper ${ }^{1}$, Hans Roeven ${ }^{1}$, Bart Janssen ${ }^{1}$

1. Thermo Fisher Scientific, Materials \& Structural Analysis, Eindhoven, the Netherlands

Structural biologists have made great progress in discovering the structures of individual proteins and protein complexes. Much of the recent progress in the structural biology field stems from advances in cryo-transmission electron microscopy (cryo-TEM), in particular single particle analysis, which has made the elucidation of (near) atomic-scale structures for proteins and protein complexes almost routine.

The key technological advances enabling this progress have been the maturation of cryo-TEM platforms (Titan Krios and Talos Arctica), the revolution in image processing software and the development of (electron counting) direct electron detectors [1].

In 2011 FEI launched the Falcon, its first commercially available direct electron detector product developed for application in 3D cryo-TEM in life sciences. For the first time a digital camera surpassed the performance (DQE) of film. This first Falcon was followed quickly by a back-thinned version (Falcon 2).

Principal to the recent success of cryo-TEM has been the introduction of the first direct electron detector that enabled electron counting and dose fractionation (Gatan K2). This development paved the way to successful application of cryo-TEM to resolve structures of small proteins [2].

Recently Thermo Fisher Scientific introduced the Falcon 3EC, a further back-thinned and faster generation of Falcon that enables electron counting on a large pixel detector. The large pixels, in combination with further back-thinning, and patented noise reduction in combination with electron counting, ensure highest signal-to-noise ratio yielding unsurpassed DQE [3].

To cope with ever increasing data rates, a new acquisition platform, Control \& Acquisition Processing Platform (CAPP), was developed. CAPP is a scalable solution for on-the-fly image processing with the possibility to include fast feedback to the microscope (figure 1). Falcon 3EC is the first camera product using the CAPP. All basic image processing (bias/gain correction, electron counting) is done on-the-fly. In addition it enables more sophisticated on-the-fly processing of which drift correction of dosefractions (including drift correction within a dose fraction) is one example that has been implemented already.

Although very important for today's data quality, this is only the beginning of what we envision to be possible using on-the-fly image processing and real-time feedback. Next generations of cameras will be built upon this platform, opening up a wealth of possibilities for the future.

In this presentation we discuss the future of direct electron detection in Cryo-TEM. Will it merely be a $\mathrm{DQE}$ race or are theoretical limits reached already? And if not DQE, what will push the structural biology field forward? Detectors have played a crucial role in the success story of cryo-TEM and we expect they will remain a key driver for ever improving resolution and faster time to results [4].

One thing that we are quite certain of is that future camera improvements will not be limited to a DQE race. 


\section{References:}

[1] Nature Methods, Special feature: Method of the Year 2015 (2015)

[2] Maofu Liao, et al., Nature 504, 107-112 (2013)

[3] M. Kuijper, et al., Journal of Structural Biology (2015)

[4] K. R. Vinothkumar and R. Henderson, Quarterly Reviews of Biophysics (2016)

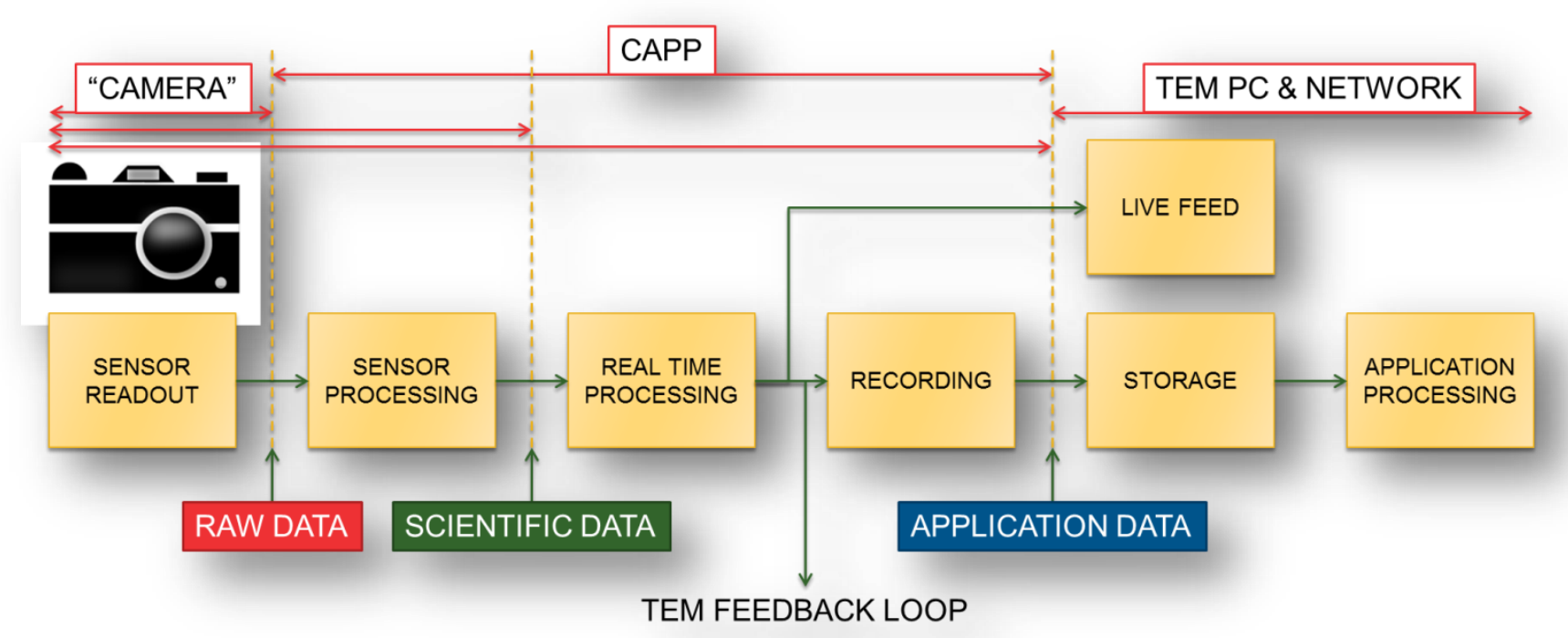

Figure 1 Describes the data path from Camera data to Application data. The Sensor delivers Raw data that is processed into meaningful Scientific data (in today's language this would be the frames or dose fractions). In the CAPP platform Scientific data can then be processed on-the-fly (currently in Falcon 3EC limited to drift correction) to yield Application data for further interpretation/processing, or it can be used to drive the microscope in real-time by the TEM feedback loop. 